\title{
Elevated serum ferritin levels in the pediatric intensive care unit
}

\author{
Sukyoung Lee, Simon J Parsons, Marinka Twilt
}

\begin{abstract}
Hemophagocytic lymphohistiocytosis (HLH) is a life-threatening inflammatory condition caused by dysregulation of the immune system. HLH can develop in children with a variety of underlying causes including genetic cause, infection, autoimmune diseases, malignancy, etc. The symptoms of HLH are often similar to other conditions such as bacterial sepsis or systemic inflammatory response syndrome. This is a problem as the similarities among those different diseases make it difficult for the doctors to diagnose HLH and this can possibly lead to a delay in treatment. $50-75 \%$ mortality is reported in patients with secondary HLH (non-inherited) who do not receive treatment. Elevated serum ferritin level, referred to as hyperferritinemia, is the most characteristic feature of HLH and may be helpful in diagnosing HLH apart from other illnesses. This research investigates the incidences of patients with elevated serum ferritin level at the pediatric intensive care unit (PICU) of Alberta Children's Hospital from 2014-2018 to gain a better understanding of HLH and hyperferritinemia. The objectives of the study are i. identify diseases associated with hyperferritinemia on the PICU; ii. predict which PICU patient with hyperferritinemia is at risk to develop HLH during PICU admission; and iii. determine mortality risk in patients with hyperferritinemia and $\mathrm{HLH}$ at the PICU. This project is a retrospective chart review. A literature review was performed on the topic of hyperferritinemia and $\mathrm{HLH}$, and relevant variables were identified for creating a Redcap database. Patient charts and medical records were examined for data collection of different elements including diagnosis, laboratory values, treatments, and survival status. Data of 91 patients who presented with hyperferritinemia in PICU from 2014 to 2018 is being examined. Although this study is currently in progress, it is anticipated to provide insight into the features associated with hyperferritinemia and determine patients with hyperferritinemia who are at risk of developing $\mathrm{HLH}$. Overall, the findings from this study may contribute to better understanding of hyperferritinemia and HLH in pediatric patients and contribute to decreasing mortality and morbidity of patients with hyperferritinemia and $\mathrm{HLH}$.
\end{abstract}

\section{Key words}

PICU, hemophagocytic lymphohistiocytosis, ferritin

Cite as: Lee S,Parsons JS, Twilt M. 2019. Elevated serum ferritin levels in the pediatric intensive care unit. Alberta Academic Review, Vol 2 (3) 19, CASCH Special Issue (not peer-reviewed), DOI: 10.29173/aar110. 